\title{
Paediatric lung transplantation
}

\author{
G.B. Mallory*, T.L. Spray ${ }^{\#}$
}

Paediatric lung transplantation. G.B. Mallory, T.L. Spray. (C) ERS Journals Ltd 2004. ABSTRACT: Since 1990, lung transplantation has been performed in infants, children and adolescents in small numbers, and the numbers, in comparison with adult transplants, remain small today. The indications for lung transplantation are similar in childhood when compared with adults, but the disease entities are distinct.

In children, severe pulmonary vascular disease is most commonly associated with developmental abnormalities or congenital heart disease, as opposed to idiopathic pulmonary hypertension. Cystic fibrosis is the dominant indication for lung transplantation in older childhood and adolescence.

The operative approach to lung transplantation in early life differs from that in adults, in that cardiopulmonary bypass is more likely to be utilised and bilateral lung transplantation is strongly preferred to single lung transplantation. Living donor lung transplantation is proportionately more common in children and adolescents than in adults. Post-transplant complications related to viral infection and post-transplant lymphoproliferative disease are more common and more likely to be severe and lifethreatening. Bronchiolitis obliterans is the most important complication after paediatric lung transplantation and limits both the quality of life and duration of survival, as in adults.

Eur Respir J 2004; 24: 839-845.
*Lung Transplant Program, Texas Children's Hospital, Houston, TX, and ${ }^{\#}$ Division of Cardiothoracic Surgery, The Children's Hospital of Philadelphia, Philadelphia, PA, USA.

Correspondence: G.B. Mallory

Texas Children's Hospital

6621 Fannin

CC 1040.00

Houston

TX 77030

USA

Fax: 18328253308

E-mail: gbmallor@texaschildrenshospital.org

Keywords: Cystic fibrosis, heart-lung transplantation, living donor lung transplantation, lung transplantation, paediatrics

Received: June 22004

Accepted: June 62004
With the pioneering work of J. Cooper and his colleagues at the University of Toronto (Toronto, ON, Canada), the era of modern lung transplantation began in the 1980s. Heartlung transplantation in children was performed in a few centres in the USA and the UK in the 1980s [1,2], but it was not until the 1990s that surgeons in selected centres in North America and Europe began to perform isolated lung transplantation in children. Subsequently, there have been a few reports documenting early success in adopting the surgical techniques, immunosuppression and post-transplant care to infants and children with reasonable outcomes [3-7].

The most recent USA data available from the United Network for Organ Sharing show that lung transplantation remains relatively limited within the paediatric age group (table 1) [8, 9]. As of May 2003, registrations for lung transplantation showed that only $4.6 \%$ of USA lung transplant candidates were $<18$ yrs old, which was comparable with the paediatric proportion for all other solid organs, except for intestinal transplants, where the majority of candidates are paediatric [8]. When the actual numbers of solid-organ transplants performed in 2001 were compared across organs, there were more than 12-times as many heart, 13-times as many kidney and 18-times as many liver transplants performed in the paediatric group than lung transplants. Even the relatively new intestinal transplantation occurred in 2.7-times as many recipients than paediatric lung recipients [8]. Since the mid 1990s, the number of heart-lung transplants performed within the USA and worldwide has continued to fall as organ blocks became less available in the USA $[8,10]$. Isolated lung or heart transplantation has been applied to clinical scenarios that heretofore led to heart-lung transplantation.

Due to the circumstances surrounding the care of patients with serious brain injuries, suitable healthy lungs are harvested at a much lower rate from brain-dead patients than other solid organs. However, it is believed that this fact, only in part, explains the low numbers of paediatric lung recipients. When the percentage of transplants performed in paediatric recipients is compared with adult recipients for each organ, paediatric lung recipients appear to be under-represented compared with most of the other organ categories. The small number of dedicated, freestanding, paediatric lung transplant centres may be a more important explanation for the relatively low number of lung transplants accomplished for infants and children in the USA. Furthermore, there are no paediatric lung transplant centres in Australia, Japan and,

Previous articles in this series: No. 1: Glanville AR, Estenne M. Indications, patient selection and timing of referral for lung transplantation. Eur Respir J 2003; 22: 845-852. No. 2: Boehler A, Estenne M. Post-transplant bronchiolitis obliterans. Eur Respir J 2003; 22: 1007-1018. No. 3: Knoop C, Haverich A, Fischer S. Immunosuppressive therapy after human lung transplantation. Eur Respir J 2004; 23: 159-171. No. 4: Kotloff RM, Ahya VN. Medical complications of lung transplantation. Eur Respir J 2004; 23: 334-342. No. 5: de Perrot M, Weder W, Patterson GA, Keshavjee S. Strategies to increase limited donor resources. Eur Respir J 2004; 23: 477-482. No. 6: Studer SM, Levy RD, McNeil K, Orens JB. Lung transplant outcomes: a review of survival, graft function, physiology, health-related quality of life and cost-effectiveness. Eur Respir J 2004; 24 : 674-685. 
Table 1.-Comparison of paediatric lung transplantation to other paediatric solid-organ transplantation in the USA from birth to 18 yrs of age in the years 2000-2002

\begin{tabular}{|c|c|c|c|c|c|c|}
\hline & \multicolumn{6}{|c|}{ Organ transplantation } \\
\hline & Lung & Heart & Liver & Kidney & Intestine & Heart-lung \\
\hline Paediatric transplant candidates listed ${ }^{\#}$ & 181 & 265 & 935 & 767 & 131 & 27 \\
\hline$\%$ of total candidates listed ${ }^{\#}$ & 4.6 & 6.6 & 5.1 & 1.4 & 42.3 & 12.1 \\
\hline Paediatric transplants performed ${ }^{\top}$ & 43 & 273 & 420 & 289 & 61 & 6 \\
\hline Free-standing paediatric transplant centres & 10 & 25 & 22 & 29 & 5 & 7 \\
\hline
\end{tabular}

Data are presented as n, unless otherwise stated. ${ }^{*}$ : from the United Network for Organ Sharing (UNOS) website for data on June 32003 [8]; : UNOS Annual Report 2001, reporting only the transplants performed in the year 2000 [9].

until recently, in Canada. In Europe, most paediatric centres appear to focus on adolescents. In 2001, the International Society for Heart and Lung Transplantation reported 61 total paediatric ( $\leqslant 17 \mathrm{yrs}$ old) lung transplants performed worldwide, of which only 18 were performed outside the USA [10]. There are only 10 certified paediatric lung transplant centres in the USA as of July 2002, and, in four of those centres, zero or one transplant were performed in each of the last 5 yrs [8]. From 1998-2002, there were only four USA centres where four or more lung or heart-lung transplant procedures were performed each year: Los Angeles Children's Hospital (Los Angeles, CA); Children's Hospital of Philadelphia (Philadelphia, PA); Children's Hospital of Pittsburgh (Pittsburgh, PA); and St. Louis Children's Hospital (St. Louis, MO). The actual peak in worldwide paediatric lung transplantation was reached in 1995-1998, with a $\sim 20 \%$ reduction in numbers since that time [10]. Thus, although paediatric lung transplantation has been established as an accepted therapy in theory, the number of transplants, patients on the waiting list, and experienced and active centres lag behind adult-lung transplantation and other paediatric solid-organ transplantation.

Heart-lung transplantation remains the preferred operation in a few adult and paediatric lung transplant centres. In 2001, 17 heart-lung transplants were performed for a primary diagnosis of cystic fibrosis (at any age) compared with 233 lung transplants worldwide; interestingly, all of these operations were performed in Europe [10].

\section{Indications}

The most common diseases in the paediatric population leading to lung transplantation in the last decade are shown in table 2 [10]. In infants, the most common indications are disorders related to pulmonary hypertension. The combination of the categories of congenital heart disease, pulmonary vascular disease and idiopathic pulmonary hypertension

Table 2. - Underlying diagnosis of paediatric lung transplant recipients transplanted worldwide, 1991-2001

\begin{tabular}{lccc}
\hline Diagnostic category & \multicolumn{3}{c}{ Age yrs } \\
\cline { 2 - 4 } & $<1$ & $1-10$ & $11-17$ \\
\hline Cystic fibrosis & 0 & 37 & 66 \\
Idiopathic pulmonary hypertension & 17 & 16 & 3 \\
Pulmonary vascular disease & 14 & 4 & 0 \\
Congenital heart disease & 33 & 11 & 9 \\
Idiopathic pulmonary fibrosis & 0 & 8 & 3 \\
Re-transplantation/graft failure & 7 & 11 & 6 \\
Other & 29 & 13 & 13 \\
\hline
\end{tabular}

Data are presented as \%. Modified from [10]. accounts for $64 \%$ of transplanted infants. Infantile pulmonary alveolar proteinosis, most commonly as a result of surfactant protein B deficiency, has become the most common indication for infantile lung transplantation in St. Louis, MO, USA [11], and would fall into the miscellaneous group. In the intermediate age group from 1-10 yrs of age, cystic fibrosis and the combined category of pulmonary vascular diseases each represent approximately one-third of the total of transplanted patients. In the 11-17 yrs age group, cystic fibrosis represents two-thirds of the total. Among the miscellaneous disease entities leading to lung transplantation at the age of $>1 \mathrm{yr}$ are: post-viral bronchiolitis obliterans, (BO) bronchopulmonary dysplasia, respiratory insufficiency (usually BO after chronic graft versus host disease) after bone marrow transplantation, and interstitial lung diseases with varying degrees of pulmonary fibrosis. Despite continuing advances in therapy, cystic fibrosis will probably remain the dominant underlying diagnosis leading to lung transplantation in older children and adolescents for the next decade.

The general criterion for lung and heart-lung transplantation in infants and children is similar to that used in adults: individuals at risk of dying from lung failure or pulmonary vascular disease and/or have a poor quality of life despite maximal medical therapy. In infants in whom organ procurement within days or weeks is currently possible in some regions of the USA, and in whom recuperation is often gratifyingly rapid, a candidate on mechanical ventilation and technological support, including nitric oxide inhalation and extracorporeal membrane oxygenation, may sometimes be appropriate for listing and transplantation [12]. Depending on the location, transplant centre and waiting list, older children are usually considered candidates if death within 6 months to 2 yrs seems likely. For children with rare diseases or atypical courses of more common diseases, the prognostication and linked listing for and timing of transplantation will depend on careful serial measurements of cardiopulmonary function to ascertain the natural history of the underlying disease process.

\section{Contraindications and psychosocial considerations}

Medical and surgical contraindications for lung transplantation in children are listed in table 3 . In the current era, most contraindications are relative. Any candidate with an active viral infection, whether it is a respiratory virus, any form of hepatitis or HIV, should not be transplanted during the infection. It should be self-evident that all candidates with cystic fibrosis have chronic and active bacterial lower respiratory infections. The vast majority of infecting organisms are not considered to be contraindications to lung transplantation. Conversely, it appears that the outcome after lung transplantation in cystic fibrosis patients infected with the most virulent and transmissible form of Burkholderia cepacia infection is poor, with a $\sim 50 \%$ risk of early mortality 
Table 3. - Medical and surgical contraindications to paediatric lung transplantation

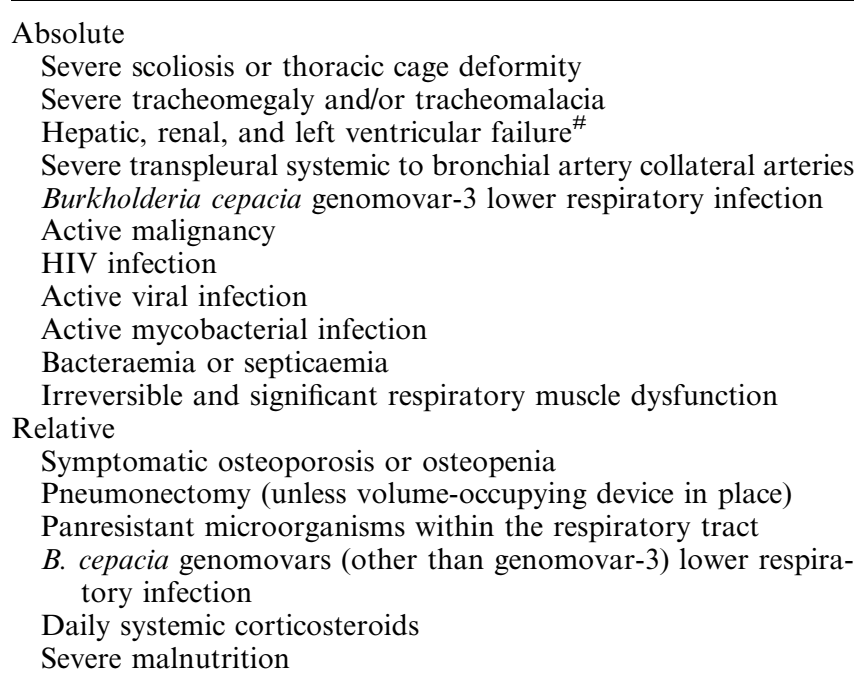

\#: multi-organ transplantation, such as liver-lung, renal-lung or heartlung transplantation, might be an appropriate option.

from infection [13, 14]. Recent microbiological research on this organism has shown that what laboratories have previously identified as $B$. cepacia should now be considered as B. cepacia complex (BCC) species [15]. Further speciation in reference laboratories can separate out the most virulent subtypes, although definitive identification of virulence factors remains to be determined. From clinical studies published to date, transplant recipients infected with genomovar-3 BCC appear to have worse outcomes when compared with individuals with other genomovars [14]. Each transplant centre has to make individual decisions based on the information available to them, with projections of how significant a given candidate's microorganisms and antibiotic susceptibility pattern might be.

Anatomical or surgical contraindications are generally centre-specific. Chemical pleurodesis increases the operative time in a somewhat unpredictable fashion, and always leads to widespread bleeding sites within the parietal pleura. Nevertheless, most transplant surgeons currently feel that candidates with pleurodesis are suitable candidates. Tracheal dilation (tracheomegaly) and/or collapsibility (tracheomalacia) make the airway anastomosis problematic and could conceivably lead to long-term mechanical ventilation. Generally, a tracheostomy alone would not be considered a contraindication, since early post-transplant decannulation could obviate the problem of a long-term portal of entry for microorganisms into the lower airway. In patients with pulmonary atresia with nonconfluent pulmonary arteries, pulmonary blood flow depends exclusively on bronchial arteries. Especially in the context of previous thoracotomy and with chronic hypoxia, there may also be diffuse arterial connections across the pleura. At St. Louis Children's Hospital, this particular clinical scenario predisposed to diffuse small arterial bleeding, which may not be manageable intra-operatively and, in fact, led to the early demise of several patients. Experience has shown that transplantation may be feasible even after repeated thoracotomies or median sternotomies.

Nonmedical contraindications include financial and psychosocial factors. Limitations in coverage and/or payment by insurers in the USA may preclude or complicate lung transplantation in a minority of individual candidates.
Community fund-raising may be necessary for many families to afford heavy uncovered costs. In view of the complexities and rigours of life after lung transplantation in any healthcare system, patients and families, the transplant team in any country, and the health insurance system must consider psychosocial issues seriously. It would seem obvious that serious mental health disorders or disabilities that would obviate cooperation with the administration of medication and/or follow-up testing are strong contraindications to transplantation. Family dysfunction, manifesting as repeated nonadherence to medical regimens in the past, should be considered a contraindication, although it may be appropriate to give an individual patient and family the opportunity to demonstrate a capacity for adherence over a defined period of time. A thorough evaluation of the child and family by a child psychologist, paediatric social worker and the team of transplant caregivers should be required before accepting or rejecting any individual candidate. Transplant centres depend on the integrity and truthfulness of referring physicians and caregivers in receiving all relevant information about patients and families that impact on past and future health behaviours. In borderline situations, probationary listing of a child, with a clear written and signed contract of expectations, may be appropriate.

There are different distribution algorithms for different countries that impact on the time of listing and waiting times. The waiting period is highly variable between individuals and between countries. Size considerations are clearly more important for paediatric recipients and donors because of the wide variability in size of the thorax and lungs during the span of childhood. Lobar transplantation and downsizing of donor lungs with a surgical stapling device are options for the transplant surgeon in the event that oversized lungs are offered. Although lungs are quite distensible, the use of undersized lungs risks overexpansion of the organ into the noncompliant portion of the lung's intrinsic pressure-volume curve and a disappointing physiological outcome.

\section{Transplant technique}

More paediatric patients, especially infants, are likely to be in the intensive care unit (ICU) at the time when organs become available than compared with adult candidates. The transition from the ICU to the operating room should be managed with careful attention to the time required, the need for line placement, and the condition and therapy of the child candidate. Many paediatric transplant surgeons elect cardiopulmonary bypass $(\mathrm{CPB})$ for all lung transplant procedures [16]. The disadvantages of CPB are the associated coagulopathy that accompanies heparinisation and the capillary leak syndrome, which affects all organs to a variable degree after CPB. The advantages of CPB include: the opportunity to deflate the native lungs to permit easier dissection of the lung and hilum, the ability to clamp and cleanse (with instillation of antibiotic) the proximal tracheobronchial airway, and the minimisation of ischaemic time.

Although single lung transplantation is a relatively common option in adult-lung transplantation, there are few paediatric recipients who have this surgery [3, 17]. Bilateral lung transplantation is the operative approach of choice for most submature patients as a result of growth considerations; for patients with cystic fibrosis because a disease with bilateral infection does not allow for single lung procedures, unless contralateral pneumonectomy is performed; and for the entire paediatric age group, since usually two lungs are available for most small patients. In most centres, heart-lung transplantation is usually reserved for patients with end-stage lung or 
pulmonary vascular disease who have irreparable congenital heart defects or left ventricular failure. Patients with pulmonary hypertension, either primary or secondary, who have right ventricular enlargement and hypertrophy will often have resolution of right heart failure and cor pulmonale after successful lung transplantation. However, there is an incidence of dynamic right ventricular outflow tract obstruction that may require beta blockade in the early postoperative period. Patients with severe pulmonary or tricuspid atrioventricular valve regurgitation or significant right heart failure in association with left ventricular dysfunction may be better served by heart and lung transplantation.

Paediatric lung transplantation is usually performed by bilateral anterolateral trans-sternal (clam-shell) thoracotomy incision. The recipient lungs are removed, and then the left and right lungs are implanted with bronchial anastomoses, using monofilament absorbable suture in a running fashion for the membranous portion of the bronchus and interrupted sutures for the cartilaginous portion. The pulmonary artery and pulmonary venous anastomoses are performed with absorbable suture, also in a running fashion, and occasionally with anastomosis to only one of the two recipient pulmonary veins if lobar transplantation is being utilised. Peribronchial tissues are tacked loosely around the anastomosis to prevent erosion of the bronchial anastomosis into the pulmonary artery, should infection or partial dehiscence occur. A broviac catheter is placed in many patients to maintain chronic vascular access in the post-transplant period.

In 1990, STARNES et al. [18] introduced living donor lung transplantation (LDLT). In the most common form of this operation, the recipient undergoes bilateral pneumonectomy, and then receives implantation of lower lobes from each of the two healthy adult donors. Given the size of even the shortest adults, LDLT is not a realistic option for children $<6$ yrs old [19]. Ideally, the donors should be taller than the recipient, so that the donor lobe can readily fill the recipient hemithorax. As a consequence of important ethical concerns, a pulmonary physician and psychologist, entirely separate from the transplant team, should evaluate all potential donors [19]. Donors should be $\geqslant 18$ yrs and in excellent health. Altruistic motive and full disclosure of the potential morbidity and mortality of elective lobectomy are mandatory. At the time of LDLT, three simultaneous thoracic operations are carried out: a requirement of resources that may be more than most transplant centres can mobilise. Although the most recent results achieved by STARNES et al. [18] in adults and children are encouraging, technical considerations have been a problem in other institutions [17]. With no end in sight to the shortage of donors, LDLT will be an option in aggressive programmes and for selected patients and families.

"Triple-drug" immunosuppression is often started prior to surgery with an oral dose of cyclosporin or tacrolimus and i.v. corticosteroids. Cyclosporin or tacrolimus is initiated promptly after surgery. In the USA, i.v. cyclosporin is usually given initially. Cyclosporin is initially titrated to trough levels $300-400 \mathrm{ng} \cdot \mathrm{mL}^{-1}$. A change to oral dosing is initiated after oral function and gastric emptying has been established. Recent insights suggest that it may be more accurate to adjust cyclosporin by using peak or concentration $2 \mathrm{~h}$ after an oral dose to optimise drug dosing [20]. Some centres have embraced tacrolimus as the calcineurin inhibitor of choice for initial immunosuppression. Initial tacrolimus dosing may be by $i . v$. drip or oral or nasogastric tube bolus dosing. Tacrolimus trough levels are titrated to $10-15 \mathrm{ng} \cdot \mathrm{mL}^{-1}$, or, initially, slightly higher in some programmes, on whole blood samples. In the past, azathioprine has been traditionally used as part of a three-drug strategy with dosing at $2-3 \mathrm{mg} \cdot \mathrm{kg}^{-1} \cdot \mathrm{day}^{-1}$ initially given $i . v$, as long as the white cell count is $>4,000$ cells $\cdot \mathrm{mm}^{-3}$. From recently published data, mycophenolate mofetil (MMF) has replaced azathioprine as the second agent in 50\% of patients transplanted after 1999 [10]. Although paediatric dosing has not been universally established, adult dosing would suggest a paediatric dose of $15 \mathrm{mg} \cdot \mathrm{kg}^{-1}$ of MMF b.i.d., either i.v. or p.o. Therapeutic drug monitoring is available for MMF dose titration and seems logical, even if empirical studies to assess efficacy are currently lacking. Corticosteroid dosing also varies by centre. In some programmes, a dose of $i$.v. methylprednisolone is given pre-transplant and continued in moderate dosing for the first 3 days post-transplant. There is broad consensus that the appropriate early dose of methylprednisolone, p.o. prednisone or prednisolone is $0.5-1.0 \mathrm{mg} \cdot \mathrm{kg}^{-1} \cdot \mathrm{day}^{-1}$. Tapering of corticosteroid dosing to $0.25-0.5 \mathrm{mg} \cdot \mathrm{kg}^{-1} \cdot \mathrm{day}^{-1}$ is common 2-3 months post-transplant. In most centres, prednisone or prednisolone q.d. is continued long term, although some programmes do consider alternate day dosing.

\section{Post-transplant considerations and complications}

Antimicrobial strategies. It is universal practice to choose an antibiotic regimen before, during, and immediately after lung transplantation that is tailored to the most likely pathogens. Initial antibiotic choice in non-cystic fibrosis patients is usually limited to a first generation cephalosporin. In cystic fibrosis patients, at least two i.v. anti-pseudomonal antibiotics are chosen, based on the most recent respiratory microbiological specimen. Other agents are chosen if Pseudomonas aeruginosa is either not present or not the exclusive pathogen. Two agents are chosen, as is the usual practice in cystic fibrosis care, to obtain synergism between antibiotics and to lessen the chance of the emergence of antibiotic-resistant organisms. In cystic fibrosis patients with a history of aspergillus infection, allergic bronchopulmonary aspergillosis or recent sputum cultures positive for Aspergillus fumigatus, antifungal therapy is initiated via the i.v. route with low-dose amphotericin B or voriconazole. Depending on clinical course, the results of respiratory culture at the time of transplant, and the first bronchoalveolar lavage culture at $24 \mathrm{~h}$ post-transplant, i.v. amphotericin B may be changed within 3-10 days to the nebulised route, usually $5-10 \mathrm{mg}$ in sterile water three-times daily for an additional 1-2 weeks. After initial parenteral therapy, transition to p.o. itraconazole or voriconazole would be appropriate.

If either the recipient or donor has a positive antibody screen for past cytomegalovirus infection, the recipient is treated with i.v. ganciclovir for at least the first week posttransplant. With the availability of the well-absorbed p.o. valganciclovir, this agent may be introduced in lieu of the $i . v$. drug as soon as the p.o. route has been established and continued for the first 2-6 months post-transplantation, depending on the centre's protocol. Trimethoprimsulfamethoxazole is commenced in the first week posttransplantation, as prophylaxis for Pneumocystis jiroveci pneumonia. Some programmes continue $P$. jiroveci pneumonia prophylaxis in the long term; others discontinue after 3 months. Oral nystatin is commonly prescribed to reduce the likelihood of clinically significant oral candidiasis. Acyclovir p.o. is used in some programmes routinely as prophylactic treatment for herpes simplex infection.

Allograft rejection. From published results in St. Louis, it appears that young children may have a lower risk of both acute and chronic rejection compared with older children and adults $[3,17]$. In a recent large series, the incidence of rejection in children $<1$ yr of age is 0.2 episodes, 0.6 episodes for those $<2$ yrs, and 1.95 episodes overall [21]. 
Nonetheless, due to the frequency of respiratory infections, timely surveillance and accurate diagnosis of allograft rejection may be even more important in the post-transplant care of children than it is in adults. The standard paediatric flexible bronchoscopes have an outer diameter of 3.4-3.8 mm and a suction channel of only $1.2 \mathrm{~mm}$. Standard biopsy forceps cannot be used through this diminutive channel. There is a mini-forceps available (FB-56D-1; Olympus America Inc., Melville, NY, USA), which can be passed through the $1.2 \mathrm{~mm}$ suction channel. Although the forceps can be used with the paediatric scopes, it is considerably more difficult, and requires many more passes of the instrument to get adequate volumes of pulmonary tissue in which to try to diagnose allograft rejection. Other techniques have been developed [22]. However, for most children aged $\geqslant 4-6$ yrs, the small adult bronchoscopes of $4.9-5.2 \mathrm{~mm}$ in outer diameter and standard biopsy forceps are used. Laryngeal mask with general anaesthesia considerably facilitates bronchoscopy in smaller and sicker patients. Standard histopathological markers of lung graft rejection are used to make the diagnosis of acute vascular rejection. Treatment of acute rejection consists of $i . v$. methylprednisolone $10 \mathrm{mg} \cdot \mathrm{kg}^{-1}$ for 3 days consecutively. For recurrent acute rejection, cytolytic therapy with an anti-lymphocyte preparation, a 6week course of once weekly p.o. methotrexate $0.15 \mathrm{mg} \cdot \mathrm{kg}^{-1}$ and/or a change in the daily immunosuppressant regimen is indicated. There have been no large retrospective comparative studies or prospective randomised studies in paediatric lung transplantation to indicate which pharmacological programme is optimal. Repeated episodes of acute graft rejection clearly predispose to chronic rejection.

Bronchiolitis obliterans syndrome (BOS) is an applicable concept for the paediatric lung transplant patient [23]. Due to the likelihood of somatic growth after lung transplantation, the definition of BOS would rely on change in the percentage of predicted values of forced expiratory volume in one second and/or forced mid-expiratory flow, as opposed to a change in absolute numbers. As a general rule, a new diagnosis of BOS would lead to augmentation of immunosuppression similar to what is employed in adults.

Somatic growth. Many transplant candidates may have chronic malnutrition and stunting of skeletal growth, as a result of the long-standing impact of the underlying disease process. Prednisone q.d., which is the rule in most paediatric lung transplant recipients in contradistinction to paediatric heart, liver and renal transplant recipients, commonly leads to a rate of skeletal growth that remains below the age-adjusted normal range. Augmentation of nutrition and correction of metabolic abnormalities are fundamental post-transplant goals. In some recipients, even when q.d. prednisone is lowered to $0.1-0.15 \mathrm{mg} \cdot \mathrm{kg}^{-1} \cdot \mathrm{day}^{-1}$, growth remains suboptimal. Administration of growth hormone in selected patients is appropriate. The growth response can be impressive, and has been reported to be both safe and effective in paediatric renal- and liver-transplant recipients [24, 25].

Nutrition. The delivery of nutrition in the immediate posttransplant period may be difficult in some patients. In patients with early graft rejection, the use of higher dose corticosteroids counteracts the goal of anabolism. In many infants, severe gastro-oesophageal reflux disease and, in older patients, gastroparesis can compromise the delivery of adequate calories by the enteral route [26]. Older patients with cystic fibrosis have a real risk for small bowel obstruction, which is a variant form of the distal intestinal obstruction syndrome. This complication can compromise transition to oral medication and nutritional rehabilitation, and may require surgery [27].
Seizures. The onset of major motor seizures in the first months after a lung transplantation is quite common [28]. Imaging studies often show reversible posterior leukoencephalopathy [29]. However, the prognosis for the majority of these patients is quite good. Phenytoin may be a particularly good choice as the anti-convulsant, since it seems to affect calcineurininhibitor hepatic drug clearance less than phenobarbitol and, perhaps, carbamazepine. In one author's experience (G.B. Mallory), most patients take phenytoin for $<3$ months and have no recurrence.

Viral infection. Children represent a risk group for particularly severe disease with certain pathogens, most notably, respiratory syncytial virus (RSV), parainfluenza virus and adenovirus, the latter of which can be particularly devastating [30]. Conversely, other viral infections, such as Epstein-Barr virus (EBV) and varicella, are known to be more benign in normal children if primary infection occurs in childhood. As a result of the higher likelihood of immunological innocence in chronically ill children and the generous and exuberant lifestyle of the companions and siblings with which infants and children tend to consort, the timely diagnosis and focused treatment of viral infections is especially important in children after a lung transplantation. Scrupulous attention to preventative measures, such as active immunisation with vaccines and consideration of passive immunisation of selected infants and very young children with palivizumab (anti-RSV immunoglobulin G), is imperative. It would be advantageous to keep these patients out of situations, such as day care, in which the likelihood of exposure to community viral pathogens is especially high. Viral infection may be associated with early graft loss and rapid development of obliterative bronchiolitis [30].

Post-transplant lymphoproliferative diseaselother neoplasias. The incidence of post-transplant lymphoproliferative disease (PTLD) seems to be higher in children than in adults [31], and there is additional data suggesting that primary EBV infection after transplantation is a related risk factor [32]. Between 1990 and 1999 in St. Louis, a total of 20\% of the cystic fibrosis lung transplant recipients developed PTLD [30], leading to high morbidity and significant mortality. Since the first measure in response to PTLD is usually a reduction in immunosuppression, survival after PTLD is complicated by a high and early incidence of BOS. Clinical scientists have developed the ability to grow clones of recipient EBV-specific cytotoxic Tlymphocytes for therapeutic use in PTLD [33]. The availability of rituximab (anti-CD20 antibody) shows early promising results, which may lead to a significant lowering in morbidity and mortality [34].

Children are at considerably lower risk of other cancers after lung transplantation because of their age. Specifically, skin, lung and gastrointestinal cancers are much rarer. EBVassociated leiomyosarcomata have been reported.

Lung function testing. Spirometry is universally employed after lung transplantation as a mainstay in assessing graft function. Children aged $\geqslant 4-6$ yrs can usually perform spirometry. There are new approaches to children somewhat younger, which may lead to reproducible flow-volume curves with forced manoeuvres in children as young as $2.5 \mathrm{yrs}$ of age [35]. Infants and younger toddlers do not have the developmental capacity for cooperating naturally with voluntary respiratory manoeuvres. In many centres, infant pulmonary function laboratories perform modified spirometry, using the rapid thoraco-abdominal compression technique under sedation. 
Lung growth. The human lung grows in response to the rhythmic application of physical stretch in a proper nutritional and hormonal milieu. It is unclear if the process of transplantation, the use of immunosuppressants or the unavoidable denervation of the lungs might stunt the growth of transplanted lungs. The growth potential of mature adult lungs or lobes transplanted into the growing thorax is also unknown. Although there is no human data to address the latter, two papers have examined lung growth in the younger patient. In St. Louis, CoHEN et al. [36] used infant lung function testing to assess airflow as a surrogate for airway calibre, and functional residual capacity as a surrogate for alveolar growth in a cohort of infants and young children aged $<3$ yrs at the time of transplantation. These physiological data support the contention that transplanted lungs grow. At the Children's Hospital of Philadelphia, Ro et al. [37] used serial chest computed tomography (CT) scans in a young population of broader age range to assess the calibre of the central intrathoracic airways over time. Their data also support the hypothesis that the airways of transplanted lungs appear to grow with somatic growth.

\section{Results}

\section{Donor statistics}

The ability to apply lung transplantation to children with cardiopulmonary disease is limited by donor availability. In St. Louis, it has been shown that the average waiting times for a patient in the USA has increased from 5.5 to 22.5 months from the early 1990 s to the start of 2000 [3, 17]. Not surprisingly, the waiting time for adolescents is now similar to the average waiting time for the adult patients in most programmes. However, there is still good reason to believe that waiting times for infants and young children tend to be considerably shorter [12].

\section{Survival statistics}

From the most recent published international data, it appears that average survival for paediatric lung transplantation (half-life: $3.5 \mathrm{yrs}$ ) falls just below that for lung transplant recipients of all ages (half-life: 3.8 yrs) [10, 38]. Interestingly, adolescents had the lowest conditional actuarial survival (5.4 yrs) and infants had the longest within the paediatric age spectrum $(7.1 \mathrm{yrs})$ [10]. In the only large series within one medical centre where paediatric and adult-lung transplant recipients with cystic fibrosis were compared, the paediatric cohort had a slightly higher survival rate at every time point after transplantation [39]. Nonetheless, there are important issues of primary viral infection, the higher incidence of PTLD and adolescent nonadherence, which may put paediatric lung transplant recipients at a greater risk of death after transplantation than their adult counterparts. A recent series of $>200$ paediatric transplants, reported from the St. Louis programme, suggests that the overall survival rate for the first year post-transplant is $77 \%$ [17]. The 3- and 5-yr survival was 63 and $54 \%$, respectively. There was no statistically significant difference between any groups of diagnoses, in terms of overall patient survival. Graft failure accounted for the majority of deaths in the first 60 days post-transplant $(56 \%)$, and infection was a relatively infrequent cause of early death $(8 \%)$. Within the group dying of graft failure in the first 60 days, re-transplant patients and recipients of LDLT were particularly common. As expected, BO was the leading cause of late deaths $(62 \%)$, with infection accounting for $22 \%$ of late deaths and malignancies $14 \%$. Interestingly, the group of patients who underwent living-donor lobar transplant had no improvement in survival over other groups, probably as a result of the higher incidence of early graft failure. However, the incidence of BO was lower in the living donor recipients. HUDDLESTON et al. [17] suggested that the acuity of illness in the living donor group was greater pretransplant than other diagnostic groups, which might account for the slightly decreased trend in survival and the lack of advantage of reduced incidence of BO. In addition, the authors showed that the ischaemic time on the transplanted lungs accounted for differences in the incidence of BO by 5 yrs post-transplant. Children who had shorter ischaemic times had a lower incidence of $\mathrm{BO}$ at $5 \mathrm{yrs}$, although the rate of $\mathrm{BO}$ development seemed to be similar in all groups of ischaemic times. Perhaps the improvement in $\mathrm{BO}$ in the living donor transplant group reflects a shorter ischaemic time, rather than an immunological advantage in these patients.

In St. Louis, age at transplant also seemed to impact the development of BO. For patients $<3$ yrs of age at the time of transplant, $28 \%$ developed BO. In contrast, $58 \%$ of patients $>3$ yrs of age at transplant ultimately developed BO in the follow-up period [17].

\section{Conclusion}

Experience has shown definitively that lung transplantation can be successfully performed in infants, children and adolescents. The indications are very similar to those in adults, although the disease entities are often different. The surgical approach is identical with the exception of a strong preference for bilateral lung transplantation and the use of cardiopulmonary bypass. Post-operative management requires special sensitivity to the issues of primary viral infection, somatic growth, scholastic progress and functioning within a family environment. As in the practice of adult-lung transplant, over time, the development of bronchiolitis obliterans in the majority limits the longevity and the quality of life in paediatric lung transplant recipients.

\section{References}

1. Starnes VA, Marshall SE, Lewiston NJ, Theodore J, Stinson EB, Shumway NE. Heart-lung transplantation in infants, children, and adolescents. J Pediatr Surg 1991; 26: 434- 438.

2. Smyth RL, Scott JP, Whitehead B, et al. Heart-lung transplantation in children. Transplant Proc 1990; 22: $1470-1471$.

3. Sweet SC, Huddleston CB, Spray TL, et al. Pediatric lung transplantation at St. Louis Children's Hospital 1990-1995. Am J Respir Crit Care Med 1997; 155: 1027-1035.

4. Metras D, Viard L, Kreitmann B, et al. Lung infections in pediatric lung transplantation: experience in 49 cases. Eur J Cardiothorac Thorac Surg 1999; 15: 490-494.

5. Aurora $\mathrm{P}$, Whitehead $\mathrm{B}$, Wade $\mathrm{A}$, et al. Lung transplantation and life extension in children with cystic fibrosis. Lancet 1999; 354: 1591-1593.

6. Starnes VA, Woo MS, MacLaughlin EF, et al. Comparison of outcomes between living donor and cadaveric lung transplantation in children. Ann Thorac Surg 1999; 68: 2279-2283.

7. Gaynor JW, Bridges ND, Clark BJ, Spray TL. Update on lung transplantation in children. Curr Opin Pediatr 1998; 10: 256-261.

8. United Network for Organ Sharing website, June 2003. www.unos.org. Date last accessed: June 32003.

9. 2001 Annual Report of the U.S. Organ Procurement and Transplantation Network and the Scientific Registry of 
Transplant Recipients: transplant data 1991-2000. Dept of Health and Human Services, Health Resources and Services Administration, Office of Special Programs, Division of Transplantation, Rockville, MD; United Network for Organ Sharing, Richmond, VA; University Renal Research and Education Association, Ann Arbor, MI.

10. Boucek MM, Edwards LB, Keck BM, et al. The registry of the International Society for Heart and Lung Transplantation: fifth official pediatric report, 2001 to 2002. J Heart Lung Transplant 2002; 21: 827-840.

11. Hamvas A, Nogee LM, Mallory GB, et al. Lung transplantation for treatment of infants with surfactant protein B deficiency. J Pediatr 1997; 130: 231-239.

12. Huddleston CB, Sweet SC, Mallory GB, Hamvas A, Mendeloff EN. Lung transplantation in very young infants. J Thorac Cardiovasc Surg 1999; 118: 796-804.

13. Aris RM, Routh JC, LiPuma J, Heath D, Gilligan PH. Lung transplantation for cystic fibrosis patients with Burkholderia cepacia complex: survival linked to genomovar type. Am J Respir Crit Care Med 2001; 164: 2102-2106.

14. Chaparro C, Maurer J, Gutierrez C, et al. Infection with Burkholderia cepacia in cystic fibrosis: outcome following lung transplantation. Am J Respir Crit Care Med 2001; 163: 43-48.

15. Mahenthiralingam E, Baldwin A, VanDamme P. Burkholderia cepacia complex infection in patients with cystic fibrosis. J Med Microbiol 2002; 51: 533-538.

16. Spray TL, Mallory GB, Canter CB, Huddleston CB. Pediatric lung transplantation: indications, techniques, and early results. J Thorac Cardiovasc Surg 1994; 107: 990-1000.

17. Huddleston CB, Bloch JB, Sweet SC, de la Morena M, Patterson GA, Mendeloff EN. Lung transplantation in children. Ann Surg 2002; 236: 270-276.

18. Starnes VA, Barr ML, Cohen RG. Lobar transplantation: indications, technique and outcome. J Thorac Cardiovasc Surg 1994; 108: 403-411.

19. Mallory GB, Cohen AH. Donor considerations in livingrelated donor lung transplantation. Clin Chest Med 1997; 18: 239-244.

20. Cole E, Midtvedt K, Johnston A, Pattison J, O'Grady C. Recommendations for the implementation of Neoral C(2) monitoring in clinical practice. Transplantation 2002; 73 : S19-S22.

21. Bridges ND, Mallory GB, Huddleston CB, Canter CE, Spray TL. Lung transplantation in infancy and early childhood. J Heart Lung Transplant 1996; 15: 895-902.

22. Mullins D, Livne M, Mallory GB, Kemp JS. A new technique for transbronchial biopsy in infants and small children. Pediatr Pulmonol 1995; 20: 253-257.

23. Estenne M, Maurer JR, Boehler A, et al. Bronchiolitis obliterans syndrome 2001: an update of the diagnostic criteria. J Heart Lung Transplant 2002; 21: 297-310.

24. Maxwell H, Rees L. Randomized controlled trial of recombinant human growth hormone in prepubertal and pubertal renal transplant recipients. Arch Dis Child 1998; 79: 481-487.
25. Rodeck B, Kardorff R, Melter M, Ehrich JH. Improvement of growth after growth hormone treatment in children who undergo liver transplantation. J Pediatr Gastroenterol Nutr 2000; 31: 286-290.

26. Berkowitz N, Schulman LL, McGregor C, Markowitz D. Gastroparesis after lung transplantation: potential role in postoperative respiratory complications. Chest 1995; 108: 1602-1607.

27. Minkes RK, Langer JC, Skinner MA, et al. Intestinal obstruction following lung transplantation in children with cystic fibrosis. J Pediatr Surg 1999; 34: 1489-1493.

28. Wong M, Mallory GB Jr, Goldstein J, Goyal M, Yamada KA. Neurological complications of pediatric lung transplantation. Neurology 1999; 53: 1542-1549.

29. Jorosz JM, Howlett DC, Cox TC, Bingham JB. Cyclosporine-related reversible posterior leukoencephalopathy following organ transplantation. Clin Neurol Neurosurg 1997; 99: 222-226.

30. Bridges ND, Spray TL, Collins M, Bowles NE, Towbin JA. Adenovirus infection in the lung results in graft failure after pediatric lung transplantation. $J$ Thorac Cardiovasc Surg 1998; 116: 617-623.

31. Cohen AH, Sweet SC, Mendeloff EN, et al. High incidence of posttransplant lymphoproliferative disease in pediatric patients with cystic fibrosis. Am J Respir Crit Care Med 2000; 161: 1252-1255.

32. Boyle GJ, Michaels MG, Webber SA, et al. Posttransplantation lymphoproliferative disorders in pediatric thoracic organ recipients. J Pediatr 1997; 131: 309-313.

33. Savoldo B, Goss J, Liu Z, et al. Generation of autologous Epstein-Barr virus-specific cytotoxic $\mathrm{T}$ cells for adoptive immunotherapy in solid organ transplant recipients. Transplantation 2001; 72: 1078-1086.

34. Reynaud-Gaubert M, Stoppa AM, Gaubert J, Thomas P, Fuentes P. Anti-CD20 monoclonal antibody therapy in Epstein-Barr Virus-associated B cell lymphoma following lung transplantation. J Heart Lung Transplant 2000; 19: 492495.

35. Vilozni D, Barker M, Jellouschek H, Heimann G, Blau H. An interactive computer-animated system (SpiroGame) facilitates spirometry in preschool children. Am J Respir Crit Care Med 2001; 164: 2200-2205.

36. Cohen AH, Mallory GB, Ross K, et al. Growth of lungs after transplantation in infants and children younger than 3 years old. Am J Respir Crit Care Med 1999; 159: 1747-1751.

37. Ro PS, Bush DM, Kramer SS, Mahboubi S, Spray TL, Bridges ND. Airway growth after pediatric lung transplantation. J Heart Lung Transplant 2001; 20: 619-624.

38. Hosenpud JD, Bennett LE, Keck BM, Boucek MM, Novick RJ. The Registry of the International Society for Heart and Lung Transplantation: eighteenth official report, 2001. J Heart Lung Transplant 2001; 20: 805-815.

39. Mendeloff EN, Huddleston CB, Mallory GB, et al. Pediatric and adult lung transplantation for cystic fibrosis. J Thorac Cardiovasc Surg 1998; 115: $404-413$. 